\title{
Spectral sum rules for the Schrödinger equation
}

\author{
Paolo Amore \\ Facultad de Ciencias, CUICBAS, Universidad de Colima, \\ Bernal Díaz del Castillo 340, Colima, Colima, Mexico \\ paolo@ucol.mx
}

August 24, 2020

\begin{abstract}
We study the sum rules of the form $Z(s)=\sum_{n} E_{n}^{-s}$, where $E_{n}$ are the eigenvalues of the time-independent Schrödinger equation (in one or more dimensions) and $s$ is a rational number for which the series converges. We have used perturbation theory to obtain an explicit formula for the sum rules up to second order in the perturbation and we have extended it nonperturbatively by means of a Padé-approximant. For the special case of a box decorated with one impurity in one dimension we have calculated the first few sum rules of integer order exactly; the sum rule of order one has also been calculated exactly for the problem of a box with two impurities. In two dimensions we have considered the case of an impurity distributed on a circle of arbitrary radius and we have calculated the exact sum rules of order two. Finally we show that exact sum rules can be obtained, in one dimension, by transforming the Schrödinger equation into the Helmholtz equation with a suitable density.
\end{abstract}

\section{Introduction}

The focus of this paper is on calculating sum rules of the form

$$
Z(s)=\sum_{n=1}^{\infty} \frac{1}{E_{n}^{s}}
$$

where $E_{n}$ are the eigenvalues of the time-independent Schrödinger equation for a given Hamiltonian $\hat{H}$ and $s$ is a rational number for which the series above converges. In particular, Sukumar [1] has discussed sum rules of the kind (11) with integer $s$ for confining potentials in one dimension, expressing them directly in terms of integrals of Green's functions. Crandall [2] has studied the case of arbitrary values $s$ by expressing the spectral zeta function (1) as a Mellin transform

$$
Z(s)=\frac{i^{s}}{\Gamma(s)} \int_{0}^{\infty} t^{s-1} \int K(x, t \mid x, 0) d x d t
$$


where $K(x, t \mid y, 0)$ is the spacetime propagator defined as

$$
K(x, t \mid y, 0)=\sum_{n=1}^{\infty} \psi_{n}(x) \psi_{n}^{\star}(y) e^{-i E_{n} t} .
$$

By using the knowledge of the exact propagator (3) for the case of a perturbed oscillator (the isotonic oscillator, Ref. [3]), $V(x)=\frac{\omega^{2} x^{2}}{2}+\frac{g}{x^{2}}$ (with $g>-1 / 8)$, Crandall was able to obtain the expressions for the corresponding spectral zeta function. Similarly, he obtained the exact expressions for sum rules of integer order, for problems where the Green's function is known explicitly, e.g. for power potentials of the form $V(x)=|x|^{\nu}$ (with $\nu>0$ ).

The main goal of the present paper is to study the case where where neither the propagator nor the Green's function are known, but the Hamiltonian can be decomposed in terms of an unperturbed Hamiltonian, for which both the eigenfunctions and eigenvalues are known, and a perturbation. Extending the approach recently introduced in refs. [4, 5], for the case of a heterogeneous Helmholtz equation, we introduce Green's functions of rational order and use them to obtain the desired sum rules in terms of suitable traces involving products of these Green's functions. Unlike in the case discussed in refs. [4, 5], however, in general the Green's function of order one cannot be calculated explicitly, being the solution of a Schwinger-Dyson equation, that can be solved perturbatively. Using perturbation theory it is possible to obtain an explicit expression for the sum rule of order $s$ (with $s$ rational number for which eq. (1) converges), even though the exact eigenvalues and wave functions for the problem are unknown.

For the special cases of impurities in one and two dimensions, for which it is possible to calculate the Green's functions exactly, we have derived exact expressions for several sum rules.

The paper is organized as follows: in Section 2 we discuss the Green's functions of rational order and explicitly obtain their expression up to second order in perturbation theory; in Section 3 the sum rule of rational order is expressed as a trace of suitable Green's function up to second order in perturbation theory; in Section 4 we discuss some applications of the formulas obtained, in one and two dimensions, comparing the analytical results with purely numerical results. Finally, in Section 5 we state our conclusions.

\section{Green's functions}

We consider the Schrödinger equation

$$
\hat{H} \Psi_{n}(x)=E_{n} \Psi_{n}(x),
$$

where $\hat{H} \equiv \hat{H}_{0}+\lambda V(x)$ is the total Hamiltonian operator and $\hat{H}_{0}$ is the unperturbed Hamiltonian, whose eigenvalues and eigenfunctions are known

$$
\hat{H}_{0} \psi_{n}(x)=\epsilon_{n} \psi_{n}(x) \text {. }
$$


Since our discussion is not limited to one-dimensional problems $n$ it understood to be the set of quantum numbers that fully identify a quantum state.

The Green's function associated to $\hat{H}_{0}$ is

$$
G_{0}(x, y)=\sum_{n} \frac{\psi_{n}(x) \psi_{n}^{\star}(y)}{\epsilon_{n}}
$$

since

$$
\hat{H}_{0} G_{0}(x, y)=\delta(x-y) .
$$

Unfortunately it is not possible to obtain a closed form for the Green's function associated to $\hat{H}$ but one can see that it obeys the Schwinger-Dyson (SD) equation

$$
G(x, y)=G_{0}(x, y)-\lambda \int G_{0}(x, z) V(z) G(z, y) d z,
$$

since

$$
\begin{aligned}
\hat{H} G(x, y) & =\hat{H}_{0} G_{0}(x, y)+\lambda V(x) G_{0}(x, y) \\
& -\lambda \int \hat{H}_{0} G_{0}(x, z) V(z) G(z, y) d z+\lambda V(x) \int G_{0}(x, z) V(z) G(z, y) d z \\
& =\delta(x-y)+\lambda V(x) G_{0}(x, y)-\lambda V(x) G(x, y) d z \\
& +\lambda V(x) \int G_{0}(x, z) V(z) G(z, y) d z \\
& =\delta(x-y)
\end{aligned}
$$

Let us now work out a spectral decomposition of $G$ in the basis of the unperturbed problem

$$
G(x, y)=\sum_{n, m} Q_{n m} \psi_{n}(x) \psi_{m}^{\star}(y) .
$$

After substituting this equation in the SD equation and projecting over the modes $\psi_{a}(x)$ and $\psi_{b}(y)$ we are left with the matrix equation

$$
Q_{a b}=\frac{\delta_{a b}}{\epsilon_{a}}-\lambda \sum_{s} \frac{\langle a|V| s\rangle}{\epsilon_{a}} Q_{s b} .
$$

Next, by assuming

$$
Q_{a b}=\sum_{j=0}^{\infty} \lambda^{j} Q_{a b}^{(j)}
$$

we can write the solution as

$$
Q_{a b}^{(j)}=\left\{\begin{array}{cc}
\frac{\delta_{a b}}{\epsilon_{a}} & , j=0 \\
-\sum_{s} \frac{\langle a|V| s\rangle}{\epsilon_{a}} Q_{s b}^{(j-1)} & , \quad j>0
\end{array}\right.
$$


The first few corrections take the form

$$
\begin{aligned}
Q_{a b}^{(1)} & =-\frac{\langle a|V| b\rangle}{\epsilon_{a} \epsilon_{b}} \\
Q_{a b}^{(2)} & =\sum_{s_{1}} \frac{\left\langle a|V| s_{1}\right\rangle\left\langle s_{1}|V| b\right\rangle}{\epsilon_{a} \epsilon_{s_{1}} \epsilon_{b}} \\
Q_{a b}^{(3)} & =-\sum_{s_{1}, s_{2}} \frac{\left\langle a|V| s_{1}\right\rangle\left\langle s_{1}|V| s_{2}\right\rangle\left\langle s_{2}|V| b\right\rangle}{\epsilon_{a} \epsilon_{s_{1}} \epsilon_{s_{2}} \epsilon_{b}}
\end{aligned}
$$

Formally one can write the solution to all orders as

$$
Q_{a b}=\left\langle a\left|\left(\mathbb{1}-\hat{H}_{0}^{-1} V+\hat{H}_{0}^{-1} V \hat{H}_{0}^{-1} V-\ldots\right) \hat{H}_{0}^{-1}\right| b\right\rangle
$$

Following Refs. 4, 5] we then define $\tilde{G}_{[1 / N]}(x, y)$ satisfying the property

$$
\int \tilde{G}_{[1 / N]}\left(x, z_{1}\right) \tilde{G}_{[1 / N]}\left(z_{1}, z_{2}\right) \ldots \tilde{G}_{[1 / N]}\left(z_{N}, y\right) d z_{1} \ldots d z_{N}=G(x, y)
$$

with $N \geq 2$.

We can decompose $\tilde{G}_{[1 / N]}$ in the basis of the unperturbed problem

$$
\tilde{G}_{[1 / N]}(x, y)=\sum_{n, m} q_{n m}^{[1 / N]} \psi_{n}(x) \psi_{m}^{\star}(y),
$$

where

$$
q_{n m}^{[1 / N]}=\iint \psi_{n}^{\star}(x) \tilde{G}_{[1 / N]}(x, y) \psi_{m}(y) d x d y .
$$

We can then use eq. (17) inside eq. (16) to obtain the matrix equation

$$
\sum_{r_{1}, \ldots, r_{N}} q_{n r_{1}}^{[1 / N]} q_{r_{1} r_{2}}^{[1 / N]} \ldots q_{r_{N} m}^{[1 / N]}=Q_{n m}
$$

From this point on we will avoid the superscript $[1 / N]$ in the coefficients whenever possible.

Next we express the coefficients as a power series in $\lambda$ as

$$
q_{n m}=\sum_{j=0}^{\infty} q_{n m}^{(j)} \lambda^{j}
$$

and substitute eqs. (12) and (20) inside eq. (19).

To order $\lambda^{0}$ we obtain the equation

$$
\sum_{r_{1} \ldots r_{N}} q_{n r_{1}}^{(0)} \ldots q_{r_{N} m}^{(0)}=\frac{\delta_{n m}}{\epsilon_{n}}
$$

whose solution is

$$
q_{n m}^{(0)}=\frac{\delta_{n m}}{\epsilon_{n}^{1 / N}} .
$$


To order $\lambda$, the l.h.s. of eq. (19) takes the form

$$
\begin{aligned}
L H S^{(1)} & =\sum_{r_{1} \ldots r_{N}}\left[q_{n r_{1}}^{(1)} q_{r_{1} r_{2}}^{(0)} \ldots q_{r_{N} m}^{(0)}+\cdots+q_{n r_{1}}^{(0)} q_{r_{1} r_{2}}^{(0)} \ldots q_{r_{N} m}^{(1)}\right] \\
& =q_{n m}^{(1)} \sum_{j=0}^{N-1} \frac{1}{\epsilon_{n}^{j / N} \epsilon_{m}^{(N-1-j) / N}} \equiv q_{n m}^{(1)} \eta_{n m}^{[1 / N]}
\end{aligned}
$$

and therefore

$$
q_{n m}^{(1)}=-\frac{1}{\eta_{n m}^{[1 / N]}} \frac{\langle n|V| m\rangle}{\epsilon_{n} \epsilon_{m}} .
$$

To order $\lambda^{2}$, the l.h.s. of eq. (19) takes the form

$$
\begin{aligned}
L H S^{(2)} & =\sum_{r_{1} \ldots r_{N}}\left[q_{n r_{1}}^{(2)} q_{r_{1} r_{2}}^{(0)} \ldots q_{r_{N} m}^{(0)}+\cdots+q_{n r_{1}}^{(0)} q_{r_{1} r_{2}}^{(0)} \ldots q_{r_{N} m}^{(2)}\right] \\
& +\sum_{r_{1} \ldots r_{N}}\left[q_{n r_{1}}^{(1)} q_{r_{1} r_{2}}^{(1)} q_{r_{2} r_{3}}^{(0)} \ldots q_{r_{N} m}^{(0)}+q_{n r_{1}}^{(1)} q_{r_{1} r_{2}}^{(0)} q_{r_{2} r_{3}}^{(1)} \ldots q_{r_{N} m}^{(0)}+\ldots\right. \\
& \left.+q_{n r_{1}}^{(0)} q_{r_{1} r_{2}}^{(0)} \ldots q_{r_{N-1} r_{N}}^{(1)} q_{r_{N} m}^{(1)}\right] \\
& =q_{n m}^{(2)} \eta_{n m}^{[1 / N]}+\sum_{r} q_{n r}^{(1)} q_{r m}^{(1)} \sum_{j=0}^{N-2} \sum_{l=0}^{N-2-j} \frac{1}{\epsilon_{n}^{j / N} \epsilon_{r}^{l / N} \epsilon_{m}^{(N-2-l-j) / N}} \\
& \equiv q_{n m}^{(2)} \eta_{n m}^{[1 / N]}+\sum_{r} q_{n r}^{(1)} q_{r m}^{(1)} \xi_{n r m}^{[1 / N]}
\end{aligned}
$$

and therefore

$$
q_{n m}^{(2)}=\frac{1}{\eta_{n m}^{[1 / N]}} \sum_{r} \frac{\langle n|V| r\rangle\langle r|V| m\rangle}{\epsilon_{n} \epsilon_{r} \epsilon_{m}}\left(1-\frac{\xi_{n r m}^{[1 / N]}}{\epsilon_{r} \eta_{n r}^{[1 / N]} \eta_{r m}^{[1 / N]}}\right) .
$$

\section{Sum rules of rational order}

The results obtained in the previous section allow us to derive an explicit expression for the sum rules of rational order.

In particular, the sum rule of order $1+1 / N$ takes the form

$$
\begin{aligned}
Z\left(1+\frac{1}{N}\right) & =\sum_{n, r} Q_{n r} q_{r n}^{[1 / N]} \\
& =\sum_{n, r} Q_{n r}^{(0)} q_{r n}^{[1 / N](0)} \\
& +\lambda \sum_{n, r}\left[Q_{n r}^{(0)} q_{r n}^{[1 / N](1)}+Q_{n r}^{(1)} q_{r n}^{[1 / N](0)}\right] \\
& +\lambda^{2} \sum_{n, r}\left[Q_{n r}^{(1)} q_{r n}^{[1 / N](1)}+Q_{n r}^{(2)} q_{r n}^{[1 / N](0)}+Q_{n r}^{(0)} q_{r n}^{[1 / N](2)}\right]+\ldots
\end{aligned}
$$


With elementary algebra we obtain

$$
\begin{aligned}
& Z^{(0)}(1+1 / N)=\sum_{n} \frac{1}{\epsilon_{n}^{1+1 / N}} \\
& Z^{(1)}(1+1 / N)=-\sum_{n}\left(1+\frac{1}{N}\right) \frac{\langle n|V| n\rangle}{\epsilon_{n}^{2+1 / N}} \\
& Z^{(2)}(1+1 / N)=-\frac{s}{2} \sum_{r, n} \frac{\epsilon_{n}^{-2-1 / N}-\epsilon_{r}^{-2-1 / N}}{\left(\epsilon_{n}-\epsilon_{r}\right)}|\langle n|V| r\rangle|^{2} .
\end{aligned}
$$

It is important to observe that the summand in the expression for $Z^{(2)}$ is finite when $n=r$ and therefore one can split the double series as $\sum_{n, r}=$ $\sum_{n=r}+\sum_{n \neq r}$; after introducing $s=1+1 / N$ one has

$$
\begin{aligned}
Z^{(2)}(s) & =\frac{s(s+1)}{2} \sum_{n} \frac{|\langle n|V| n\rangle|^{2}}{\epsilon_{n}^{2+s}} \\
& -\frac{s}{2} \sum_{r \neq n} \frac{\epsilon_{n}^{-1-s}-\epsilon_{r}^{-1-s}}{\left(\epsilon_{n}-\epsilon_{r}\right)}|\langle n|V| r\rangle|^{2} .
\end{aligned}
$$

However

$$
\frac{\epsilon_{n}^{-1-s}-\epsilon_{r}^{-1-s}}{\epsilon_{n}-\epsilon_{r}}=\left(\epsilon_{n}^{-2-s}+\epsilon_{r}^{-2-s}\right)+\frac{\epsilon_{r} \epsilon_{n}^{-s-2}-\epsilon_{n} \epsilon_{r}^{-s-2}}{\epsilon_{n}-\epsilon_{r}},
$$

and

$$
\begin{aligned}
-\frac{s}{2} & \sum_{r \neq n} \frac{\epsilon_{n}^{-1-s}-\epsilon_{r}^{-1-s}}{\left(\epsilon_{n}-\epsilon_{r}\right)}|\langle n|V| r\rangle|^{2}=-s \sum_{r \neq n} \epsilon_{n}^{-2-s}|\langle n|V| r\rangle|^{2} \\
& -\frac{s}{2} \sum_{r \neq n} \frac{\epsilon_{r} \epsilon_{n}^{-s-2}-\epsilon_{n} \epsilon_{r}^{-s-2}}{\epsilon_{n}-\epsilon_{r}}|\langle n|V| r\rangle|^{2} .
\end{aligned}
$$

The first contribution can be simplified using the completeness of the basis

$$
\begin{aligned}
-s \sum_{r \neq n} \epsilon_{n}^{-2-s}|\langle n|V| r\rangle|^{2} & =-s \sum_{n} \epsilon_{n}^{-2-s}\left\langle n\left|V\left[\sum_{r}|r\rangle\langle r|-| n\rangle\langle n|\right] V\right| n\right\rangle \\
& =-s \sum_{n} \epsilon_{n}^{-2-s}\left(\left\langle n\left|V^{2}\right| n\right\rangle-\langle n|V| n\rangle^{2}\right) .
\end{aligned}
$$

Finally, after these manipulations, the second order correction to the sum rule takes the form

$$
\begin{aligned}
Z^{(2)}(s) & =\frac{s(s+3)}{2} \sum_{n} \frac{|\langle n|V| n\rangle|^{2}}{\epsilon_{n}^{2+s}}-s \sum_{n} \frac{\left\langle n\left|V^{2}\right| n\right\rangle}{\epsilon_{n}^{2+s}} \\
& -\frac{s}{2} \sum_{r \neq n} \frac{\epsilon_{r} \epsilon_{n}^{-s-2}-\epsilon_{n} \epsilon_{r}^{-s-2}}{\epsilon_{n}-\epsilon_{r}}|\langle n|V| r\rangle|^{2},
\end{aligned}
$$


and the sum rule of order $s$ reads

$$
\begin{aligned}
Z(s) & =\sum_{n} \frac{1}{\epsilon_{n}^{s}}-\lambda \sum_{n} s \frac{\langle n|V| n\rangle}{\epsilon_{n}^{1+s}} \\
& +\lambda^{2}\left[\frac{s(s+3)}{2} \sum_{n} \frac{|\langle n|V| n\rangle|^{2}}{\epsilon_{n}^{2+s}}-s \sum_{n} \frac{\left\langle n\left|V^{2}\right| n\right\rangle}{\epsilon_{n}^{2+s}}\right. \\
& \left.-\frac{s}{2} \sum_{r \neq n} \frac{\epsilon_{r} \epsilon_{n}^{-s-2}-\epsilon_{n} \epsilon_{r}^{-s-2}}{\epsilon_{n}-\epsilon_{r}}|\langle n|V| r\rangle|^{2}\right]+\ldots
\end{aligned}
$$

We can obtain a non-perturbative extension of the expression above by introducing the simple Padé approximant

$$
Z^{(\text {Pade })}(s)=\frac{Z^{(0)}(s)+\lambda \frac{\left(Z^{(1)}(s)\right)^{2}-Z^{(0)}(s) Z^{(2)}(s)}{Z^{(1)}(s)}}{1-\lambda \frac{Z^{(2)}(s)}{Z^{(1)}(s)}}
$$

with a pole at $\lambda Z^{(2)}(s)=Z^{(1)}(s)$. A pole in the sum rules naturally occurs when one eigenvalue gets arbitrarily close to zero and the sum rules diverges.

\section{Applications}

In the following we will discuss the application of eq. (34) to the case of a linear potential in a one-dimensional box and the calculation of exact sum rules of integer order for special cases where the Green's functions can be known explicitly.

\subsection{Linear potential in a box}

In this case the unperturbed Hamiltonian is the Hamiltonian of a particle in

a one-dimensional box of size $L$ and the perturbation is represented by the potential

$$
V(x)=\left\{\begin{array}{ll}
\kappa x & , \quad|x|<L / 2 \\
\infty & , \quad|x| \geq L / 2
\end{array} .\right.
$$

It is convenient to introduce the dimensionless variable $y=x / L(|y| \leq 1 / 2)$ and cast the Schrödinger equation into a dimensionless form as

$$
\left[-\frac{1}{2} \frac{d^{2}}{d y^{2}}+\rho y\right] \phi_{n}(y)=\tilde{E}_{n} \phi_{n}(y)
$$

where

$$
\rho \equiv \kappa \frac{M L^{3}}{\hbar^{2}} \quad, \quad \tilde{E}_{n} \equiv E_{n} \frac{M L^{2}}{\hbar^{2}}
$$


and

$$
\phi_{n}(y) \equiv \psi_{n}(y L)
$$

From eq. (37) we see that we can work with the unperturbed problem corresponding to a box of unit length, $L=1$, and setting $\hbar=M=1$.

The matrix elements of the potential in the unperturbed basis are then

$$
\begin{aligned}
& \langle m|V| n\rangle=\left\{\begin{array}{cc}
0 & , \quad m=n \\
\frac{4 m n \rho\left((-1)^{m+n}-1\right)}{\pi^{2}\left(m^{2}-n^{2}\right)^{2}}, & m \neq n
\end{array}\right. \\
& \left\langle m\left|V^{2}\right| n\right\rangle=\left\{\begin{array}{cc}
\frac{\left(\pi^{2} n^{2}-6\right) \rho^{2}}{2^{2 \pi^{2} n^{2}}} \quad, \quad m=n \\
\frac{4 m n \rho^{2}\left((-1)^{m+n}+1\right)}{\pi^{2}(m-n)^{2}(m+n)^{2}}, & m \neq n
\end{array}\right.
\end{aligned}
$$

The spectral sum rule will then read

$$
\begin{aligned}
Z(s) & =\left(\frac{\hbar^{2}}{M L^{2}}\right)^{-s}\left[2^{s} \pi^{-2 s}\left(\zeta(2 s)-\frac{s \rho^{2} \zeta(2(2+s))}{3 \pi^{4}}+\frac{2 s \rho^{2} \zeta(2(3+s))}{\pi^{6}}\right)\right. \\
& \left.+\rho^{2} 2^{s+6} \pi^{-2(s+4)} s \sum_{n \neq r} \frac{\left((-1)^{n+r+1}+1\right)^{2}\left(n^{4} r^{-2 s-2}-r^{4} n^{-2 s-2}\right)}{\left(n^{2}-r^{2}\right)^{5}}\right] \\
& +O\left(\rho^{4}\right)
\end{aligned}
$$

To test this result we have applied the Rayleigh-Ritz method with 2000 eigenfunctions and we have numerically calculated the lowest eigenvalues of eq. (37) for $\rho=k / 500$, with $k=0, \ldots, 500$. With these eigenvalues we can approximately calculate the sum rules as

$$
Z(s)=\left(\frac{\hbar^{2}}{M L^{2}}\right)^{-s}\left[\sum_{n=1}^{N} \frac{1}{\tilde{E}_{n}^{s}}+\sum_{n=N+1}^{\infty} \frac{1}{\left(\tilde{E}_{n}^{(W K B)}\right)^{s}}\right],
$$

where $\tilde{E}_{n}$ are the numerical eigenvalues calculated using the Rayleigh-Ritz method and $\tilde{E}_{n}^{(W K B)}$ are the approximations obtained using the WKB method

$$
\begin{aligned}
\tilde{E}_{n}^{(W K B)} & =\frac{\pi^{2}(2 n+1)^{2}}{8}+\frac{\rho^{2}}{6(2 \pi n+\pi)^{2}} \\
& +\frac{2 \rho^{4}}{9(2 \pi n+\pi)^{6}}+\frac{8 \rho^{6}}{9(2 \pi n+\pi)^{10}}+\ldots .
\end{aligned}
$$

In our calculation we have used $N=500$ because typically the accuracy of the RR eigenvalues decreases as $n$ increases. For a given value of $s$ we have then calculated the numerical sum rules at the different values of $\rho$ mentioned earlier and we have used these results to obtain a fit as a function of $\rho$. 
For instance, for $s=1$ we have

$$
\begin{aligned}
Z^{(f i t)}(1) & =\left(\frac{\hbar^{2}}{M L^{2}}\right)^{-1}\left[0.333333+0.0000881836 \rho^{2}+3.25102 \cdot 10^{-8} \rho^{4}\right. \\
& \left.+9.99546 \cdot 10^{-10} \rho^{6}-3.80688 \cdot 10^{-10} \rho^{8}+\ldots\right]
\end{aligned}
$$

which should be compared with the exact result of eq. (41), to order $\rho^{2}$

$$
\begin{aligned}
Z(1) & =\left(\frac{\hbar^{2}}{M L^{2}}\right)^{-1}\left[\frac{1}{3}-\frac{4 \rho^{2}}{14175}+0.0003703703703 \rho^{2}+\ldots\right] \\
& \approx\left(\frac{\hbar^{2}}{M L^{2}}\right)^{-1}\left[0.333333+0.0000881834 \rho^{2}+\ldots\right] .
\end{aligned}
$$

Similarly, for $s=3 / 4$ we have

$$
\begin{aligned}
Z^{(f i t)}(3 / 4) & =\left(\frac{\hbar^{2}}{M L^{2}}\right)^{-3 / 4}\left[0.789007+0.0000975667 \rho^{2}+3.09362 \cdot 10^{-8} \rho^{4}\right. \\
& \left.+1.15761 \cdot 10^{-9} \rho^{6}-4.46889 \cdot 10^{-10} \rho^{8}+\ldots\right]
\end{aligned}
$$

which should be compared with the exact result of eq. (41), to order $\rho^{2}$

$$
\begin{aligned}
Z(3 / 4) & =\left(\frac{\hbar^{2}}{M L^{2}}\right)^{-1}\left[\frac{2^{3 / 4} \zeta\left(\frac{3}{2}\right)}{\pi^{3 / 2}}-\frac{\rho^{2}\left(\pi^{2} \zeta\left(\frac{11}{2}\right)-6 \zeta\left(\frac{15}{2}\right)\right)}{2 \sqrt[4]{2} \pi^{15 / 2}}+\ldots\right] \\
& \approx\left(\frac{\hbar^{2}}{M L^{2}}\right)^{-1}\left[0.789011+0.0000975665 \rho^{2}+\ldots\right]
\end{aligned}
$$

In the present case we cannot apply the diagonal Padé approximant of eq. (35) because the spectral sum rule is an even function of $\rho$.

\subsection{Infinite box decorated with impurities}

The unperturbed Hamiltonian is unchanged with respect to the previous example, while the perturbation is represented by the potential

$$
V(x)=\kappa \delta(x-a),
$$

with $|a|<L / 2$.

In this special case it is possible to solve exactly the Schwinger-Dyson equation (8), as noticed in Refs. 2, 6], and the Green's function takes the form

$$
G(x, y)=G_{0}(x, y)-\frac{\kappa G_{0}(x, a) G_{0}(a, y)}{1+\kappa G_{0}(a, a)} .
$$

As before it is convenient to cast the time-independent Schrödinger equation in a dimensionless form as

$$
\left[-\frac{1}{2} \frac{d^{2}}{d \bar{y}^{2}}+\rho \delta(\bar{y}-\bar{a})\right] \phi_{n}(\bar{y})=\tilde{E}_{n} \phi_{n}(\bar{y})
$$


where the definitions of $\tilde{E}_{n}$ and $\phi_{n}(\bar{y})$ are unchanged while

$$
\rho \equiv \kappa \frac{M L}{\hbar^{2}} \quad, \quad \bar{q} \equiv q / L .
$$

The spectral sum rules can then be written as

$$
Z(s)=\sum_{n} \frac{1}{E_{n}^{s}}=\Gamma^{s} \sum_{n} \frac{1}{\tilde{E}_{n}^{s}} \equiv \Gamma^{s} \bar{Z}(s)
$$

where $\Gamma \equiv M L^{2} / \hbar^{2}$ has dimensions of inverse energy.

In the dimensionless form, the unperturbed Green's function takes the form [7]

$$
G_{0}(\bar{x}, \bar{y})=\frac{1}{2}[-(2 \bar{x}-1)(2 \bar{y}+1) \theta(\bar{x}-\bar{y})-(2 \bar{x}+1)(2 \bar{y}-1) \theta(\bar{y}-\bar{x})],
$$

and the full Green's function is obtained from eq. (49).

In terms of this Green's function we can easily obtain the sum rule of order one, exact to all orders as

$$
\begin{aligned}
Z(1) & =\frac{M L^{2}}{\hbar^{2}} \int_{-1 / 2}^{1 / 2} G(\bar{x}, \bar{x}) d \bar{x} \\
& =\frac{M L^{2}}{\hbar^{2}} \frac{4+\rho-16 \rho \bar{a}^{4}}{6\left(2+\rho-4 \rho \bar{a}^{2}\right)},
\end{aligned}
$$

with a pole at

$$
\rho_{c}=-\frac{2}{1-4 \bar{a}^{2}},
$$

corresponding to a critical coupling

$$
\kappa_{c}=-\frac{2 \hbar^{2}}{M L\left(1-4 a^{2} / L^{2}\right)} .
$$

At $\kappa=\kappa_{c}$ the sum rule diverges because a bound state with null energy appears; as soon as $\kappa<\kappa_{c}$ the energy of the bound state decreases and the sum rule passes from being positive to being negative. The absence of further poles signals that the potential supports only a single bound state. One should also observe that, if we keep the other parameters fixed, but we let $L \rightarrow \infty$, the critical value $\kappa_{c}$ approaches 0 from below, consistent with the well known result that the attractive delta potential always supports a bound state regardless of how small is the coupling.

For a system with $\rho<\rho_{c}$, the bound state can disappear as a result of any of the following actions: by making the attractive potential weaker (i.e. making $\kappa$ smaller), by moving the walls of the box closer (i.e. making $L$ smaller) or by moving the impurity closer to one of the walls.

Additionally we note that at

$$
\rho_{0}=-\frac{4}{1-16 \bar{a}^{4}}
$$




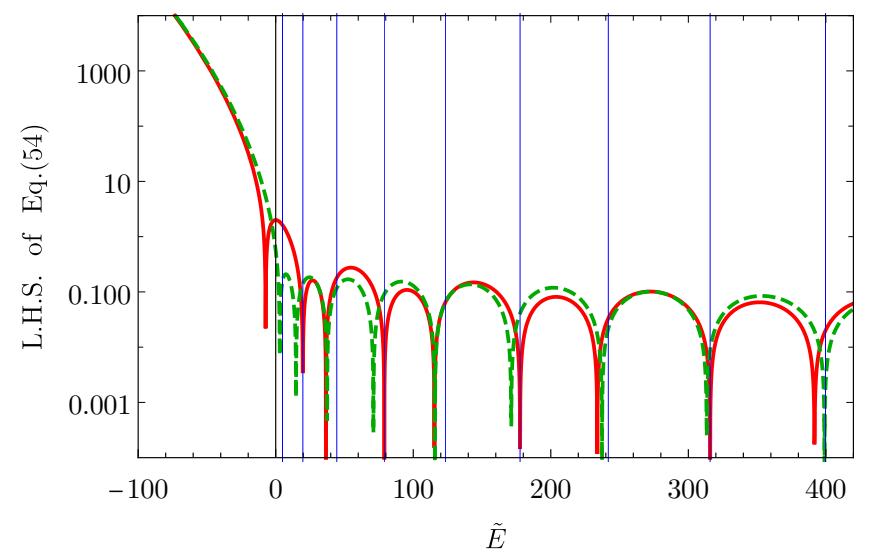

Figure 1: LHS of eq. (58) as a function of $\tilde{E}$, for $\rho=-4$; the solid and dashed curves correspond to $\bar{a}=0$ and $\bar{a}=0.4$, respectively. The thin vertical lines correspond to the dimensionless eigenvalues of the particle in a box, $\tilde{E}=n^{2} \pi^{2} / 2$. Observe that for $\bar{a}=0$ the odd states are unaffected by the Dirac delta.

the sum rule vanishes. This root is lost when $1-16 \bar{a}^{4}=0$, or $\bar{a}= \pm 1 / 2$. In this case the Dirac delta falls on the border of the box and therefore its effect disappears.

If we solve the time-independent Schrödinger equation for this problem exactly we see that the dimensionless eigenvalues are solutions to the transcendental equation

$$
\rho \frac{-\cos \sqrt{2 \tilde{E}}+\cos (\bar{a} \sqrt{8 \tilde{E}})}{2 \tilde{E}}+\frac{\sin \sqrt{2 \tilde{E}}}{\sqrt{2 \tilde{E}}}=0 .
$$

The critical value of $\rho$ can be obtained from this equation by solving for $\rho$ and letting $\tilde{E} \rightarrow 0$; in this case we obtain eq. (55). The l.h.s. of eq. (58) is plotted in Fig. 1 for $\rho=-4$, where the solid and dashed curves correspond to $\bar{a}=0$ and $\bar{a}=0.4$, respectively.

We have verified eq. (54) by calculating numerically the first 2000 roots of eq. (58) with an accuracy of 100 digits, for $\rho=-4$ and $\bar{a}=0$. In this case the sum rule is approximated as

$$
\bar{Z}^{(\mathrm{num})}(1)=\sum_{n=1}^{2000} \frac{1}{\tilde{E}_{n}^{\text {(num })}} \approx-0.0001013 .
$$

A much better estimate can be obtained by taking into account the asymptotic behavior of the eigenvalues

$$
\tilde{E}_{n} \approx \tilde{E}_{n}^{(\text {asym })} \equiv \frac{n^{2} \pi^{2}}{2}+\sum_{j=1}^{\infty} c_{j}(n) \rho^{j},
$$


where

$$
\begin{aligned}
c_{1}(n) & =1+(-1)^{1-n} \cos (2 n \pi \bar{a}) \\
c_{2}(n) & =-\frac{3}{4 n^{2} \pi^{2}}+\frac{(-1)^{n} \cos (2 n \pi \bar{a})}{n^{2} \pi^{2}}-\frac{\cos (4 n \pi \bar{a})}{4 n^{2} \pi^{2}} \\
& +\frac{2(-1)^{n} \bar{a} \sin (2 n \pi \bar{a})}{n \pi}-\frac{\bar{a} \sin (4 n \pi \bar{a})}{n \pi} \\
c_{3}(n) & =\frac{5}{2 n^{4} \pi^{4}}-\frac{1}{3 n^{2} \pi^{2}} \\
& +\frac{(-1)^{n} \cos (2 n \pi \bar{a})\left(-30+n^{2} \pi^{2}\left(3+20 \bar{a}^{2}\right)\right)}{8 n^{4} \pi^{4}} \\
& -\frac{\cos (4 n \pi \bar{a})\left(-3+8 n^{2} \pi^{2} \bar{a}^{2}\right)}{2 n^{4} \pi^{4}} \\
+ & \frac{(-1)^{n} \cos (6 n \pi \bar{a})\left(-6+n^{2} \pi^{2}\left(-1+36 \bar{a}^{2}\right)\right)}{24 n^{4} \pi^{4}} \\
& -\frac{5(-1)^{n} \bar{a} \sin (2 n \pi \bar{a})}{n^{3} \pi^{3}}+\frac{4 \bar{a} \sin (4 n \pi \bar{a})}{n^{3} \pi^{3}}+\frac{(-1)^{1+n} \bar{a} \sin (6 n \pi \bar{a})}{n^{3} \pi^{3}}
\end{aligned}
$$

Additionally one should observe that for $\bar{a}=0$, the odd eigenfunctions are unaffected by the Dirac delta function and therefore $\tilde{E}_{2 n}=\frac{(2 n)^{2} \pi^{2}}{2}$. In this case we can approximate the sum rule as

$$
\begin{aligned}
\bar{Z}^{(\text {num })}(1) & =\sum_{n=1}^{1000} \frac{1}{\tilde{E}_{2 n-1}}+\sum_{n=1001}^{\infty} \frac{1}{\tilde{E}_{2 n-1}^{(\text {asym })}}+\sum_{n=1}^{\infty} \frac{1}{\tilde{E}_{2 n}} \\
& \approx 1.44 \cdot 10^{-25}
\end{aligned}
$$

where we have used the coefficients of eq. (61).

Let us now modify the unperturbed Hamiltonian of the box with a single impurity by adding a constant term $\gamma$

$$
\hat{H}_{0}=-\frac{\hbar^{2}}{2 m} \frac{d^{2}}{d x^{2}}+\gamma
$$

The corresponding Green's function can be easily calculated and it reads

$$
G_{0}(\bar{x}, \bar{y})=\frac{\operatorname{csch}(\sqrt{2 \gamma})}{\sqrt{2 \gamma}}(-\cosh (\sqrt{2 \gamma}(\bar{x}+\bar{y}))+\cosh (\sqrt{2 \gamma}(1-|\bar{x}-\bar{y}|)))
$$

The Green's function for the full problem is obtained as before using eq. (49). By introducing the dimensionless parameter

$$
\bar{\gamma} \equiv \frac{\gamma M L^{2}}{\hbar^{2}}
$$




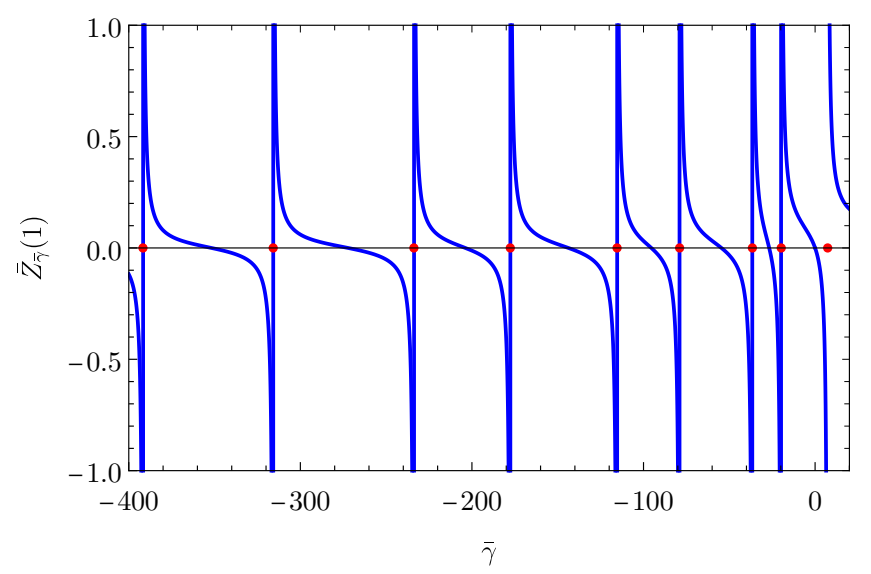

Figure 2: $\bar{Z}_{\bar{\gamma}}(1)$ for $\bar{a}=0$ using $\rho=-4$ as a function of $\gamma$. The red points are the numerical solution of eq. (58).

we can calculate the sum rule

$$
Z_{\bar{\gamma}}(1)=\frac{M L^{2}}{\hbar^{2}} \bar{Z}_{\bar{\gamma}}(1)=\frac{M L^{2}}{\hbar^{2}} \frac{\mathcal{N}_{\bar{\gamma}}}{\mathcal{D}_{\bar{\gamma}}}
$$

where

$$
\begin{aligned}
\mathcal{N}_{\bar{\gamma}} & \equiv(-1+\rho) \sqrt{\bar{\gamma}}+\sqrt{2} \operatorname{coth}(\sqrt{2 \bar{\gamma}})(-\rho+\bar{\gamma}) \\
& +\rho \operatorname{csch}(\sqrt{2 \bar{\gamma}})\left(\sqrt{2} \cosh \left((2 \bar{\gamma})^{3 / 2}\right)-2 \bar{\gamma}^{3 / 2} \sinh \left((2 \bar{\gamma})^{3 / 2}\right)\right) \\
\mathcal{D}_{\bar{\gamma}} & \equiv \sqrt{2} \rho\left(\cosh (\sqrt{2 \bar{\gamma}})-\cosh \left((2 \bar{\gamma})^{3 / 2}\right)\right) \operatorname{csch}(\sqrt{2 \bar{\gamma}}) \bar{\gamma}+2 \bar{\gamma}^{3 / 2}
\end{aligned}
$$

As we can see from Fig. 2 the sum rule has poles at $\gamma=-\tilde{\epsilon}_{n}$, where $\tilde{\epsilon}_{n}$ is an exact eigenvalue (in dimensionless form) of the full Hamiltonian. This can be understood since

$$
\bar{Z}_{\bar{\gamma}}(1)=\sum_{n=1}^{\infty} \frac{1}{\tilde{\epsilon}_{n}+\bar{\gamma}}
$$

We can use the sum rule above to calculate

$$
\bar{Z}(j)=\sum_{n} \frac{1}{\tilde{\epsilon}_{n}^{j}}
$$

as

$$
\bar{Z}(j)=\frac{(-1)^{j-1}}{(j-1) !} \lim _{\bar{\gamma} \rightarrow 0} \frac{d^{j-1}}{d \bar{\gamma}^{j-1}} \bar{Z}_{\bar{\gamma}}(1)
$$


In particular

$$
\begin{aligned}
\bar{Z}(2) & =\frac{1}{180\left(2+\rho-4 \rho \bar{a}^{2}\right)^{2}}[32 \\
& +\rho^{2}\left(1+16 \bar{a}^{2}-160 \bar{a}^{4}+256 \bar{a}^{6}+256 \bar{a}^{8}\right) \\
& \left.+\rho\left(8+16 \bar{a}^{2}\left(6-40 \bar{a}^{2}+32 \bar{a}^{4}\right)\right)\right] \\
\bar{Z}(3) & =\frac{1}{3780\left(2+\rho-4 \rho \bar{a}^{2}\right)^{3}}[256 \\
& +\rho\left(78+1440 \bar{a}^{2}-9408 \bar{a}^{4}+10752 \bar{a}^{6}-4608 \bar{a}^{8}\right) \\
& +\rho^{2}\left(12+480 \bar{a}^{2}-3456 \bar{a}^{4}+27648 \bar{a}^{8}-24576 \bar{a}^{10}\right) \\
& \left.+\rho^{3}\left(1+48 \bar{a}^{2}-432 \bar{a}^{4}+6912 \bar{a}^{8}-12288 \bar{a}^{10}-4096 \bar{a}^{12}\right)\right] \\
\bar{Z}(4) & =\frac{1}{226800\left(2+\rho-4 \rho \bar{a}^{2}\right)^{4}}[6144 \\
& +64 \rho\left(37+756 \bar{a}^{2}-4960 \bar{a}^{4}+6272 \bar{a}^{6}-3840 \bar{a}^{8}+1024 \bar{a}^{10}\right) \\
& +16 \rho^{2}\left(-1+4 \bar{a}^{2}\right)^{2}\left(27+1600 \bar{a}^{2}+4704 \bar{a}^{4}-10752 \bar{a}^{6}+5888 \bar{a}^{8}\right) \\
& +48 \rho^{3}\left(-1+4 \bar{a}^{2}\right)^{3}\left(-1-96 \bar{a}^{2}-672 \bar{a}^{4}+768 \bar{a}^{8}\right) \\
& \left.+3 \rho^{4}\left(-1+4 \bar{a}^{2}\right)^{4}\left(1+112 \bar{a}^{2}+1120 \bar{a}^{4}+1792 \bar{a}^{6}+256 \bar{a}^{8}\right)\right]
\end{aligned}
$$

Let us now test the accuracy of eq. (54). As we have seen, when the delta potential becomes sufficiently attractive, a bound state with arbitrarily small energy appears and the sum rules above diverge. In particular, for $\bar{a}=0$, this corresponds to $\rho=-2$.

If we apply the perturbative formulas for $s=1$ and $\bar{a}=0$ the approximate expression for the (dimensionless) sum rule is

$$
\bar{Z}(1)=0.333333-0.0833333 \lambda \rho+0.0416456 \lambda^{2} \rho^{2}
$$

and the corresponding Pade approximant of eq. (35) reads

$$
\bar{Z}^{\text {Pade }}(1)=\frac{0.333333+0.0832489 \lambda \rho}{1+0.499747 \lambda \rho}
$$

with a pole at $\rho \approx-2.00101$, remarkably close to the exact value 1 . Unfortunately the pole of the Padé approximant moves to $\rho \approx-1.412$ for the sum rule of order 2, and even worse results are found for $s=3$ and higher. However, the pole of the sum rules, which provides the critical coupling at which a bound state appears, does not depend on the order of the sum rule, whereas the pole in the Padé approximant depends on $s$; it is easy to understand why the simple Padé of eq. (35) works so well for the sum rule of order one: in that case, the exact sum rule takes the form of a diagonal $[1,1]$ Padé, which is precisely the form in eq. (35). The sum rules of higher orders, are still diagonal Padé, but of

\footnotetext{
${ }^{1} \lambda$ is just a book-keeping parameter which should be set to 1 at the end of the calculation.
} 
orders $[2,2],[3,3]$, etc. In those cases, a reliable approximation of the pole would require perturbative calculations of higher order or a different implementation of the Padé approximant.

Let us now consider the case of two Dirac delta located at $x=a$ and $x=b$. The time-independent Schrödinger equation in dimensionless form will read

$$
\left[-\frac{1}{2} \frac{d^{2}}{d \bar{y}^{2}}+\rho \delta(\bar{y}-\bar{a})+\mu \delta(\bar{y}-\bar{b})\right] \phi_{n}(\bar{y})=\tilde{E}_{n} \phi_{n}(\bar{y})
$$

The Green's function for the case of two or more impurities can also be constructed explicitly, as recently done in Ref. [8]. The final form is

$$
\begin{aligned}
G(x, y) & =G_{0}(x, y)-\frac{1}{D}\left\{-\rho G_{0}(x, a) G_{0}(a, y)-\mu G_{0}(x, b) G_{0}(b, y)\right. \\
& +\mu \rho\left[G_{0}(x, a)\left(G_{0}(b, y) G_{0}(a, b)-G_{0}(b, b) G_{0}(a, y)\right)\right. \\
& \left.\left.+G_{0}(x, b)\left(G_{0}(a, y) G_{0}(b, a)-G_{0}(a, a) G_{0}(b, y)\right)\right]\right\}
\end{aligned}
$$

where

$$
D \equiv\left(1+\rho G_{0}(a, a)\right)\left(1+\mu G_{0}(a, a)\right)-\mu \rho G_{0}(a, b) G_{0}(b, a)
$$

Our formula does not reproduce completely eq. (9) of Ref. 8 , because of the a sign difference in our definitions of Green's function (which amounts to change the couplings $(\rho, \mu) \rightarrow(-\rho,-\mu))$ and in two typos in the formula in Ref. [8].

Also in this case we can easily calculate the sum rule of order one to all orders

$$
Z(1)=\frac{M L^{2}}{\hbar^{2}}[\mathcal{F}(\bar{a}, \bar{b}, \rho, \mu) \theta(a-b)+\mathcal{F}(\bar{b}, \bar{a}, \mu, \rho) \theta(-a+b)]
$$

where

$$
\mathcal{F}(a, b, \rho, \mu)=\frac{\mathcal{N}(\bar{a}, \bar{b}, \rho, \mu)}{\mathcal{D}(\bar{a}, \bar{b}, \rho, \mu)}
$$

and

$$
\begin{aligned}
\mathcal{N}(\bar{a}, \bar{b}, \rho, \mu) & \equiv 4+\rho\left(1-16 \bar{a}^{4}\right)+\mu\left(1-16 \bar{b}^{4}\right) \\
& -2 \mu \rho(-1+2 \bar{a})(\bar{a}-\bar{b})(1+2 \bar{b}) \\
& \cdot\left(1+4\left(\bar{a}^{2}+\bar{b}^{2}-\bar{a} \bar{b}\right)+2(\bar{b}-\bar{a})\right) \\
\mathcal{D}(a, b, \rho, \mu) & \equiv 12+6 \rho\left(1-4 \bar{a}^{2}\right)+6 \mu\left(1-4 \bar{b}^{2}\right) \\
& -12 \mu \rho(-1+2 \bar{a})(\bar{a}-\bar{b})(1+2 \bar{b})
\end{aligned}
$$

Notice that for $\bar{b}=\bar{a}$, eq. (76) correctly reduces to eq. (54) for a potential $(\rho+\mu) \delta(x-\bar{a})$.

In Figure 3 we plot $\bar{Z}(1)$ for $\bar{a}=-\bar{b}=1 / 6$ using $\mu=\rho$ (left plot) and $\mu=-\rho$ (right plot). The solid lines are the exact results of eq. (76), whereas 

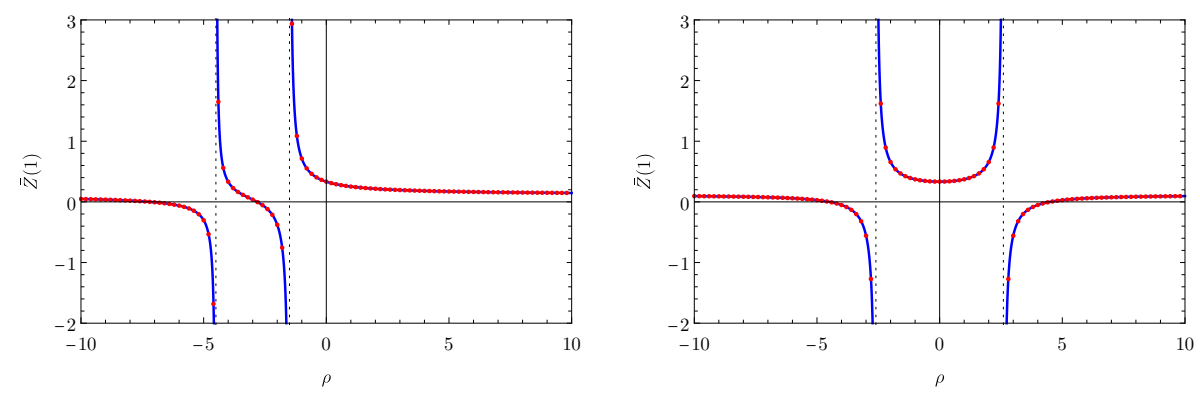

Figure 3: $\bar{Z}(1)$ for $\bar{a}=-\bar{b}=1 / 6$ using $\mu=\rho$ (left plot) and $\mu=-\rho$ (right plot). The solid lines are the exact results of eq. (76), whereas the dots are the numerical results obtained with the Rayleigh-Ritz method with 2000 basis elements.

the dots are the numerical results obtained with the Rayleigh-Ritz method with 2000 basis elements. For the case of the right plot, which corresponds to $\mu=-\rho$ and $\bar{b}=-\bar{a}$, the sum rule is an even function of $\rho$ and there is only one bound state (the two singularities merely correspond to configurations that are one the reflection of the other). For $\mu=\rho$, one the other hand, the sum rule has two singularities, located at $\rho_{1}=-(1-2 \bar{a})^{-1}$ and $\rho_{2}=-(2 \bar{a}(1-2 \bar{a}))^{-1}$ : the second singularity corresponds to the critical value of $\rho$ at which a second bound state appears.

\subsection{Two dimensional regions decorated with impurities}

Consider a circle of radius $R$ and Dirichlet boundary conditions at the border; the Green's function obeys the equation

$$
-\frac{\hbar^{2}}{2 M} \Delta G_{0}-E_{\gamma} G_{0}=\delta^{(2)}\left(\mathbf{r}-\mathbf{r}^{\prime}\right)
$$

As usual it is convenient to write this equation in a dimensionless form by introducing the definitions:

$$
\begin{aligned}
\bar{r} & \equiv \frac{r}{R} \\
\bar{\gamma} & \equiv \frac{2 M R^{2} E_{\gamma}}{\hbar^{2}} \\
\bar{G}_{0} & \equiv \frac{\hbar^{2}}{2 M R^{2}} G_{0}
\end{aligned}
$$

and the corresponding equation becomes

$$
-\Delta \bar{G}_{0}-\bar{\gamma} \bar{G}_{0}=\delta^{(2)}\left(\overline{\mathbf{r}}-\overline{\mathbf{r}}^{\prime}\right)
$$


The Green's function satisfying the equation above is [9]

$$
\begin{aligned}
\bar{G}_{0}\left(\bar{r}, \theta, \bar{r}^{\prime}, \theta^{\prime}\right) & =-\frac{1}{4} \sum_{n=-\infty}^{\infty} \cos \left[n\left(\theta-\theta^{\prime}\right)\right] \\
& \times J_{n}\left(\bar{k}_{0} \bar{r}_{<}\right)\left[Y_{n}\left(\bar{k}_{0} \bar{r}_{>}\right)-\frac{Y_{n}\left(\bar{k}_{0}\right)}{J_{n}\left(\bar{k}_{0}\right)} J_{n}\left(\bar{k}_{0} \bar{r}_{>}\right)\right]
\end{aligned}
$$

where

$$
\bar{k}_{0} \equiv \sqrt{\bar{\gamma}} \quad, \quad \bar{r}_{>} \equiv \max \left(\bar{r}, \bar{r}^{\prime}\right) \quad, \quad \bar{r}_{<} \equiv \min \left(\bar{r}, \bar{r}^{\prime}\right)
$$

We can cast the Green's function in a more compact form as

$$
\bar{G}_{0}\left(\bar{r}, \theta, \bar{r}^{\prime}, \theta^{\prime}\right)=\sum_{n=0}^{\infty} g_{n}\left(\bar{r}, \bar{r}^{\prime}\right) \cos \left[n\left(\theta-\theta^{\prime}\right)\right]
$$

where

$$
\begin{aligned}
& \bar{g}_{0}\left(\bar{r}, \bar{r}^{\prime}\right)=-\frac{1}{4} J_{0}\left(\bar{k}_{0} \bar{r}_{<}\right)\left[Y_{0}\left(\bar{k}_{0} \bar{r}_{>}\right)-\frac{Y_{0}\left(\bar{k}_{0}\right)}{J_{0}\left(\bar{k}_{0}\right)} J_{0}\left(\bar{k}_{0} \bar{r}_{>}\right)\right] \\
& \bar{g}_{n}\left(\bar{r}, \bar{r}^{\prime}\right)=-\frac{1}{2} J_{n}\left(\bar{k}_{0} \bar{r}_{<}\right)\left[Y_{n}\left(\bar{k}_{0} \bar{r}_{>}\right)-\frac{Y_{n}\left(\bar{k}_{0}\right)}{J_{n}\left(\bar{k}_{0}\right)} J_{n}\left(\bar{k}_{0} \bar{r}_{>}\right)\right] \quad, \quad n \geq 1
\end{aligned}
$$

We may be tempted to generalize our previous discussion for the onedimensional delta function to the two-dimensional case, but in doing so we would immediately stumble into a problem: the formal solution for the Green's function in eq. (49) is spoiled by the short distance behavior of the two-dimensional unperturbed Green's function

$$
\lim _{\vec{\eta} \rightarrow 0} G_{0}(\vec{r}, \vec{r}+\vec{\eta})=\infty
$$

The two-dimensional Dirac delta potential is an example of quantum mechanical problem where renormalization is needed [10, 11, 12, 13, 14.

Following Ref. [10] we consider the potential

$$
V(r)=\kappa \frac{\delta\left(r-r_{0}\right)}{2 \pi r}
$$

and the corresponding Hamiltonian

$$
\hat{H}=-\frac{\hbar^{2}}{2 M} \Delta+\kappa \frac{\delta\left(r-r_{0}\right)}{2 \pi r}
$$

The Green's function associated to this Hamiltonian obeys the equation

$$
-\Delta \bar{G}-\bar{\gamma} \bar{G}+\frac{\rho}{2 \pi \bar{r}} \delta\left(\bar{r}-\bar{r}_{0}\right) \bar{G}=\delta^{(2)}\left(\mathbf{r}-\mathbf{r}^{\prime}\right)
$$

where $\rho \equiv 2 M \kappa / \hbar$. 
The Dyson-Schwinger equation for the Green's function can be expressed in terms of the integrals

$$
\mathcal{I}_{k+1}\left(\bar{r}, \theta, \bar{r}^{\prime}, \theta^{\prime}\right) \equiv-\int_{0}^{1} \int_{0}^{2 \pi} \mathcal{I}_{k}\left(\bar{r}, \theta, \bar{r}_{1}, \theta_{1}\right) \bar{V}\left(\bar{r}_{1}\right) \bar{G}_{0}\left(\bar{r}_{1}, \theta_{1}, \bar{r}^{\prime}, \theta^{\prime}\right) r_{1} d r_{1} d \theta_{1}
$$

with

$$
\begin{aligned}
\mathcal{I}_{1}\left(\bar{r}, \theta, \bar{r}^{\prime}, \theta^{\prime}\right) & \equiv \int_{0}^{1} \int_{0}^{2 \pi} \bar{G}_{0}\left(\bar{r}, \theta, \bar{r}_{1}, \theta_{1}\right) \bar{V}\left(\bar{r}_{1}\right) \bar{G}_{0}\left(\bar{r}_{1}, \theta_{1}, \bar{r}^{\prime}, \theta^{\prime}\right) r_{1} d r_{1} d \theta_{1} \\
& =\frac{\rho}{2} \sum_{n=0}^{\infty}\left(1+\delta_{n 0}\right) \cos \left[n\left(\theta-\theta^{\prime}\right)\right] g_{n}\left(\bar{r}, \bar{r}_{0}\right) g_{n}\left(\bar{r}_{0}, \bar{r}^{\prime}\right)
\end{aligned}
$$

It is easy to see that

$$
\begin{aligned}
\mathcal{I}_{k}\left(\bar{r}, \theta, \bar{r}^{\prime}, \theta^{\prime}\right) & =(-1)^{k+1}\left(\frac{\rho}{2}\right)^{k} \\
& \times \sum_{n=0}^{\infty}\left(1+\delta_{n 0}\right)^{k} \cos \left[n\left(\theta-\theta^{\prime}\right)\right] g_{n}\left(\bar{r}, \bar{r}_{0}\right)\left[g_{n}\left(\bar{r}_{0}, \bar{r}_{0}\right)\right]^{k-1} g_{n}\left(\bar{r}_{0}, \bar{r}^{\prime}\right)
\end{aligned}
$$

Finally, the full Green's function reads

$$
\begin{aligned}
\bar{G}\left(\bar{r}, \theta, \bar{r}^{\prime}, \theta^{\prime}\right) & =\bar{G}_{0}\left(\bar{r}, \theta, \bar{r}^{\prime}, \theta^{\prime}\right)-\sum_{k=1}^{\infty} \mathcal{I}_{k}\left(\bar{r}, \theta, \bar{r}^{\prime}, \theta^{\prime}\right) \\
& =\sum_{n=0}^{\infty} \tilde{g}_{n}\left(\bar{r}, \bar{r}^{\prime}\right) \cos \left[n\left(\theta-\theta^{\prime}\right)\right]
\end{aligned}
$$

where

$$
\tilde{g}_{n}\left(\bar{r}, \bar{r}^{\prime}\right)=\left\{g_{n}\left(\bar{r}, \bar{r}^{\prime}\right)-\left(\frac{\rho}{2}\right) \frac{\left(1+\delta_{n 0}\right) g_{n}\left(\bar{r}, \bar{r}_{0}\right) g_{n}\left(\bar{r}_{0}, \bar{r}^{\prime}\right)}{1+\frac{\rho}{2}\left(1+\delta_{n 0}\right) g_{n}\left(\bar{r}_{0}, \bar{r}_{0}\right)}\right\}
$$

The sum rule of order 2 can be calculated by extracting the zero-energy Green's function of order two, expanding the energy dependent Green's function in $\bar{\gamma}$ and selecting the linear contribution, which is then used to calculate the corresponding trace:

$$
Z(2)=\sum_{n=0}^{\infty} z_{n}(2)
$$


where

$$
\begin{aligned}
z_{0}(2) & =\frac{1}{64\left(\rho \log \left(r_{0}\right)-2 \pi\right)^{2}}\left[4 \rho^{2}\left(r_{0}^{2}-1\right)^{2}-2 \pi \rho\left(11 r_{0}^{4}-16 r_{0}^{2}+5\right)\right. \\
& +\rho \log \left(r_{0}\right)\left(5 \rho+r_{0}^{4}\left(-5 \rho+4 \rho \log \left(r_{0}\right)+24 \pi\right)+2 \rho \log \left(r_{0}\right)-8 \pi\right) \\
& \left.+8 \pi^{2}\right] \\
z_{1}(2) & =\frac{1}{96\left(\rho-\rho r_{0}^{2}+4 \pi\right)^{2}}\left[(\rho+4 \pi)^{2}-16 \rho(\rho+4 \pi) r_{0}^{2}\right. \\
& +\rho r_{0}^{4}\left(31 \rho+2 r_{0}^{2}\left(-9 \rho+\rho r_{0}^{2}+20 \pi\right)\right. \\
& \left.\left.+48 \log \left(r_{0}\right)\left(\rho \log \left(r_{0}\right)-4 \pi\right)+16 \pi\right)\right] \\
z_{2}(2) & =\frac{1}{288\left(\rho-\rho r_{0}^{4}+8 \pi\right)^{2}}\left[(\rho+8 \pi)^{2}\right. \\
& +\rho r_{0}^{4}\left(64(\rho+6 \pi)+2 \rho r_{0}^{8}-128(\rho+4 \pi) r_{0}^{2}\right. \\
& \left.\left.+r_{0}^{4}\left(61 \rho-72 \rho \log \left(r_{0}\right)+112 \pi\right)+72(\rho+8 \pi) \log \left(r_{0}\right)\right)\right] \\
& \ldots
\end{aligned}
$$

A direct inspection of the above expressions shows the presence of poles at

$$
\begin{aligned}
\rho_{c}^{(0)} & =\frac{2 \pi}{\log r_{0}} \\
\rho_{c}^{(j)} & =\frac{4 \pi j}{1-r_{0}^{2 j}} \quad, \quad j=1,2, \ldots
\end{aligned}
$$

corresponding to the critical couplings at which one of the eigenvalues vanishes.

We can easily verify this result for the ground state by writing explicitly the zero energy wave function

$$
\Psi_{0}(r)=N\left[-\theta\left(r-r_{0}\right) \log r-\theta\left(r_{0}-r\right) \log r_{0}\right]
$$

where $N$ is the normalization constant (irrelevant for our calculation).

By substituting this expression inside the Schrodinger equation we obtain

$$
-\Delta \Psi_{0}(r)+\frac{\rho}{2 \pi r} \delta\left(r-r_{0}\right) \Psi_{0}(r)=\frac{\delta\left(r-r_{0}\right)}{r_{0}}\left(1-\frac{\log r_{0} \rho}{2 \pi}\right)=0
$$

whose solution provides the critical coupling $\rho_{c}^{(0)}$.

Similarly, for the states with non-vanishing angular momentum $(m>0)$, the zero-energy solutions are

$$
\Psi_{m}(r)=N_{m}\left[\theta\left(r-r_{0}\right) \sinh (m \log r)+\theta\left(r_{0}-r\right) \frac{1}{2} r^{m}\left(1-r_{0}^{-2 m}\right)\right]
$$

and the corresponding Schrodinger equation is

$$
\begin{aligned}
-\Delta \Psi_{m}(r) & +\frac{m^{2}}{r^{2}} \Psi_{m}(r)+\frac{\rho}{2 \pi r} \delta\left(r-r_{0}\right) \Psi_{m}(r) \\
& =\frac{r_{0}{ }^{-m-1} \delta\left(r-r_{0}\right)\left(\rho\left(r_{0}{ }^{2 m}-1\right)-4 \pi m\right)}{4 \pi}=0
\end{aligned}
$$


whose solution provides the critical coupling $\rho_{c}^{(m)}$

For $0<r_{0} \ll 1$ and keeping $\rho$ fixed, the sum rule behaves as

$$
\begin{aligned}
Z(2) & =\left(\frac{1}{32}+\frac{5 \rho-8 \pi}{64 \rho \log \left(r_{0}\right)}+\ldots\right)+\left(\frac{1}{96}-\frac{7 \rho r_{0}^{2}}{48(\rho+4 \pi)}+\ldots\right) \\
& +\left(\frac{1}{288}+\ldots\right)+\left(\frac{1}{640}+\ldots\right)+\left(\frac{1}{1200}+\ldots\right)+\ldots
\end{aligned}
$$

where the leading contributions have the general form $\frac{\left(1-\delta_{n 0} / 2\right)}{8(n+1)^{2}(n+2)}$ (see also eq. (5.18) of Ref. [15]).

Notice that

$$
\sum_{n=0}^{\infty} \frac{\left(1-\delta_{n 0} / 2\right)}{8(n+1)^{2}(n+2)}=\frac{\pi^{2}}{48}-\frac{5}{32}
$$

is the sum rule of order two for the unit circle [16].

As a result in this limit we have

$$
Z(2) \approx \frac{\pi^{2}}{48}-\frac{5}{32}+\frac{5 \rho-8 \pi}{64 \rho \log \left(r_{0}\right)}-\frac{7 \rho r_{0}^{2}}{48(\rho+4 \pi)}+\ldots
$$

\subsection{Simple harmonic oscillator with an anharmonic per- turbation}

The Schrödinger equation in this case is

$$
\left[-\frac{\hbar^{2}}{2 M} \frac{d^{2}}{d x^{2}}+\frac{1}{2} M \omega^{2} x^{2}+g x^{4}\right] \Psi_{n}(x)=E_{n} \Psi_{n}(x),
$$

and it can be cast in the dimensionless form

$$
\left[-\frac{1}{2} \frac{d^{2}}{d y^{2}}+\frac{1}{2} y^{2}+\rho y^{4}\right] \Phi_{n}(y)=\tilde{E}_{n} \Phi_{n}(y)
$$

where $y=x \sqrt{\frac{M \omega}{\hbar}}$ and

$$
\rho \equiv \frac{\hbar g}{M^{2} \omega^{3}} \quad, \quad \tilde{E}_{n} \equiv \frac{E_{n}}{\hbar \omega} .
$$

Observing that the WKB approximation tells us that $\tilde{E}_{n} \approx n^{4 / 3}$ for $n \rightarrow \infty$, we conclude that $Z(1)$ is finite. However the first term in eq. (34) diverges at $s=1$ because of the behavior of the eigenvalues of the simple harmonic oscillator, $\tilde{\epsilon}_{n}=n+1 / 2$.

The situation worsens for the first order correction in eq. (34): in this case for $n \gg 1$ the summand behaves as

$$
\frac{\langle n|V| n\rangle}{\epsilon_{n}^{1+s}} \propto \frac{1}{n^{-1+s}},
$$


due to the matrix element

$$
\left\langle n\left|y^{4}\right| n\right\rangle=\frac{3}{4}\left(2 n^{2}+2 n+1\right),
$$

and the series converges for $s>2$. A similar analysis for the diagonal contribution to second order reveals the corresponding series converges for $s>3$.

The origin of these problems lies in the fact that we are not describing correctly the asymptotic behavior of the spectrum and therefore the expansion of the sum rule breaks down at some finite order, no matter how large $s$ is. If $s$ is sufficiently large, however, and one sums only the first few orders of the expansion, the sum rule receives most of its contributions from the low part of the spectrum, that can be well described in the SHO basis. In this case we expect to obtain a good approximation from our perturbative formula 2 .

\subsection{Transforming the Schrödinger equation into the Helmholtz equation}

Consider the time independent Schrödinger equation in one dimension

$$
-\frac{\hbar^{2}}{2 m} \frac{d^{2} \psi}{d x^{2}}+V(x) \psi(x)=E \psi(x)
$$

where $V(x)$ is a potential and $\psi(x)$ obeys Dirichlet boundary conditions at $x= \pm L / 2$.

Similarly we consider the Helmholtz equation for a heterogeneous system

$$
-\frac{d^{2} \phi}{d u^{2}}=E \Sigma(u) \phi(u)
$$

where $\Sigma(u)$ is density and $\phi(u)$ obey Dirichlet boundary conditions at $u= \pm \ell / 2$.

We assume

$$
\phi(u)=R(u) \psi(x(u)),
$$

and substitute it inside eq. (109) obtaining

$$
-\frac{x^{\prime}(u)^{2}}{\Sigma(u)} \frac{d^{2} \psi}{d x^{2}}-\left(\frac{x^{\prime \prime}(u)}{\Sigma(u)}+\frac{2 R^{\prime}(u) x^{\prime}(u)}{R(u) \Sigma(u)}\right) \frac{d \psi}{d x}-\frac{R^{\prime \prime}(u)}{R(u) \Sigma(u)} \psi(x)=E \psi(x) .
$$

Eq. (111) takes the form of a time independent Schrödinger equation in one dimension provided that

$$
\begin{aligned}
& \frac{x^{\prime}(u)^{2}}{\Sigma(u)}=\frac{\hbar^{2}}{2 m} \\
& \frac{x^{\prime \prime}(u)}{\Sigma(u)}+\frac{2 R^{\prime}(u) x^{\prime}(u)}{R(u) \Sigma(u)}=0,
\end{aligned}
$$

\footnotetext{
${ }^{2}$ An alternative approach would consist of working with an unperturbed basis with eigenvalues that grow faster that $4 / 3$, such as for a box with hard walls; in this case the expansion is well-defined and one could improve the accuracy of the calculation by enlarging the size of the box and including higher order corrections.
} 
with a potential

$$
V(x)=\frac{R^{\prime \prime}(u(x))}{R(u(x)) \Sigma(u(x))} .
$$

The first equation requires

$$
x(u)=\frac{\hbar}{\sqrt{2 m}} \int \sqrt{\Sigma(u)} d u+c_{1} .
$$

With the substitution of $x(u)$ in the second equation we obtain

$$
\frac{\Sigma^{\prime}(u)}{\Sigma(u)}+4 \frac{R^{\prime}(u)}{R(u)}=0
$$

which has the solution

$$
R(u)=\frac{c_{2}}{\Sigma^{1 / 4}(u)} .
$$

The potential is

$$
V(x)=-\frac{5 \Sigma^{\prime}(u)^{2}}{16 \Sigma(u)^{3}}+\frac{\Sigma^{\prime \prime}(u)}{4 \Sigma(u)^{2}} .
$$

Notice that the condition $V(x)=0$, corresponding to a free particle trapped in an infinite well, implies a differential equation for the density of the string with general solution (originally found by Borg in [17])

$$
\Sigma(u)=\frac{\beta}{(1+\alpha u)^{4}} .
$$

As an example, consider the density

$$
\Sigma(u)=\frac{\beta}{(1+\alpha u)^{2}},
$$

from which

$$
\begin{aligned}
& x(u)=c_{1}+\frac{\sqrt{\beta} \hbar \log (\alpha u+1)}{\sqrt{2} \alpha \sqrt{M}} \\
& R(u)=c_{2} \beta^{-1 / 4} \sqrt{\alpha u+1},
\end{aligned}
$$

and

$$
V(x)=\frac{\alpha^{2}}{4 \beta}
$$

corresponding to a particle in a box, with a constant potential $\alpha^{2} / 4 \beta$.

The sum rule calculated from the eigenvalues of the Schrodinger equation reads

$$
\begin{aligned}
Z^{S c h}(1) & =\sum_{n=1}^{\infty} \frac{1}{\frac{\hbar^{2} \pi^{2} n^{2}}{2 M L^{2}}+\frac{\alpha^{2}}{4 \beta}} \\
& =\frac{\sqrt{2} \sqrt{\beta} L \sqrt{M}}{\alpha \hbar} \operatorname{coth}\left(\frac{\alpha L \sqrt{M}}{\sqrt{2} \sqrt{\beta} \hbar}\right)-\frac{2 \beta}{\alpha^{2}} .
\end{aligned}
$$


The same sum rule can be calculated using the Helmholtz equation (eq.(11) of Ref. [7])

$$
\begin{aligned}
Z^{H e l m h o l t z}(1) & =\int_{-\ell / 2}^{\ell / 2}\left(\frac{\ell}{4}-\frac{u^{2}}{\ell}\right) \Sigma(u) d u \\
& =\frac{4 \beta}{\alpha^{3} \ell} \operatorname{arctanh}\left(\frac{\alpha \ell}{2}\right)-\frac{2 \beta}{\alpha^{2}} .
\end{aligned}
$$

The two expressions are seen to be equivalent after relating $L$ to $\ell$ through the equation

$$
L=x(\ell / 2)-x(-\ell / 2)=\frac{\sqrt{2} \sqrt{\beta} \hbar}{\alpha \sqrt{M}} \operatorname{arctanh}\left(\frac{\alpha \ell}{2}\right) .
$$

In a similar way one can calculate higher order sum rules either directly from the eigenvalues of the Schrodinger equation or using the trace formulas discussed in Ref. [7.

\section{Conclusions}

We have discussed the calculation of sum rules $Z(s)=\sum_{n=1}^{\infty} \frac{1}{E_{n}^{s}}$ where $E_{n}$ are the eigenvalues of the time-independent Schrödinger equation in one or more dimensions and $n$ is the set of quantum numbers identifying a given state. The sum rule converges for $s>s_{0}$, where the value of $s_{0}$ depends both on the potential and on the dimensionality of the problem.

Extending the method of Refs. 4, [5] we have obtained an explicit formula for the sum rule of order $s$ to second order in perturbation theory. We have applied this formula to a simple problem (linear potential in a box) and we have compared the exact results with precise numerical results obtained applying the Rayleigh-Ritz method, reproducing the latter with great accuracy.

For the special case of a infinite box decorated with an impurity at its interior, we have obtained the exact sum rules for the first few integer orders exploiting the possibility of obtaining the Green's function for this problem exactly to all orders. The sum rule has been tested numerically for a set of parameters, solving the transcendental equation for the first 2000 eigenvalues numerically with great precision and then completing the series using the asymptotic behavior of the eigenvalues.

In two dimensions we have considered a disk, with a impurity distributed on a circle, centered at the origin, and we have calculated the spectral sum rule of order two exactly. For a fixed size of the impurity and letting the strength of the potential change, one observes that the sum rule has an infinite number of poles which provide the critical couplings where the energy of a state of given angular momentum vanishes.

Finally, we have discussed a different strategy for calculating the sum rules, which is based on the transformation of the one-dimensional Schrödinger equation into a Helmholtz equation for an heterogeneous medium. 


\section{Acknowledgements}

The research of P.A. was supported by the Sistema Nacional de Investigadores (México). The author would like to thank Dr. F.M.Fernández for useful comments and suggestions.

\section{References}

[1] Sukumar, C. V. "Greens functions and a hierarchy of sum rules for the eigenvalues of confining potentials." American Journal of Physics 58.6 (1990): 561-565.

[2] Crandall, Richard E. "On the quantum zeta function." Journal of Physics A: Mathematical and General 29.21 (1996): 6795.

[3] Weissman, Yitzhak, and Joshua Jortner. "The isotonic oscillator." Physics Letters A 70.3 (1979): 177-179.

[4] Amore, Paolo, "On the calculation of exact sum rules of rational order for quantum billiards" (2019)

[5] Amore, Paolo, "On the calculation of exact sum rules of rational order for quantum billiards (spectrum with a null eigenvalue)" (2019)

[6] Glasser, M. L., and L. M. Nieto. "The energy level structure of a variety of one-dimensional confining potentials and the effects of a local singular perturbation." Canadian Journal of Physics 93.12 (2015): 1588-1596.

[7] Amore, Paolo. "Exact sum rules for inhomogeneous strings." Annals of Physics 338 (2013): 341-360.

[8] Glasser, M. L. "A note on the Exact Green function for a quantum system." Frontiers in Physics 7 (2019): 7.

[9] Duffy, Dean G. Green's functions with applications. Chapman and Hall/CRC, 2015.

[10] Mead, Lawrence R., and John Godines. "An analytical example of renormalization in twodimensional quantum mechanics." American Journal of Physics 59.10 (1991): 935-937.

[11] Gosdzinsky, P., and Rolf Tarrach. "Learning quantum field theory from elementary quantum mechanics." American Journal of Physics 59.1 (1991): 70-74.

[12] Jackiw, R. "MAB Beg Memorial Volume." Diverse Topics in Theoretical and Mathematical Physics (1991): 35. 
[13] Bender, Carl M., and Lawrence R. Mead. "Dimensional expansion for the delta-function potential." European journal of physics 20.2 (1999): 117.

[14] Holstein, Barry R. "Understanding an anomaly." American Journal of Physics 82.6 (2014): 591-596.

[15] Steiner, Frank. "Spectral Sum Rules for the Circular AharonovBohm Quantum Billiard." Fortschritte der Physik/Progress of Physics 35.1 (1987): 87-114.

[16] Amore, Paolo. "Exact sum rules for inhomogeneous drums." Annals of Physics 336 (2013): 223-244.

[17] G. Borg, Acta Mathematica $78 \quad$ (1946) 196. doi:10.1007/BF02421600. 